\title{
Robust PID Control of the Quadrotor Helicopter *
}

\author{
R.A. García, F.R. Rubio and M.G. Ortega \\ Dpto Ing Sistemas y Automática. Universidad de sevilla, Escuela \\ Técnica Superior de Ingeniería, 41092, Sevilla, Spain. \\ $\{$ ramongr, rubio, mortega\}@us.es
}

\begin{abstract}
In this paper a robust PID control strategy via affine parametrization is designed for an multivariable nonlinear unmanned aerial vehicle. The robustness of the controlled system is assured by using the $H_{\infty}$ norm of the weighted complementary sensitivity function. Simulation results carried out using a complete nonlinear model are shown, wherein the performance achieved with this control strategy is shown.
\end{abstract}

Keywords: PID, Robust control, UAV, Simulation, Model.

\section{INTRODUCTION}

Unmanned Aerial Vehicles, UAVs, are well advanced in recent years, especially the quadrotor equipments. They are helicopters whose main feature is that they have four coplanar motors. Furthermore, as helicopter, it enables a hover, allowing the testing of control techniques for stabilization and height regulation regardless of the displacement in the XY plane.

This kind of airship also requires continuous control action, because the system is unstable. The slightest change in an uncontrolled action from the engines can cause the system leave the stable operating point.

A lot of researches have been carried out in a wide range of fields in control and robotics, such as computer vision feedback, fusion sensor, and linear and nonlinear control methodologies to improve performance of this kind of system.

Equations of movements for this kind of helicopter can be found in (Castillo et al (2007), Raffo et al (2008)). These equations can be used for the control of the helicopter by means of many control strategies, such as $\mathrm{MPC}, H_{\infty}$, and PID, among others (Alexis et al (2011), Chen et al (2003,AIAA), Chen et al (2003)). Feedback information can be provided by inertial measurement units, GPS, or even computer vision systems (Altug et al (2002)).

In this paper, a fixed-structure robust PIDs are used to control both the attitude and the height of a quadrotor helicopter. PIDs are very extended in industry due to the possibility of experimental settings and the insight of their parameters tuning. Focusing on robust control, this control theory allows to consider uncertainties which are not described in a nominal model, such as delays or variations in certain values due to complex measurements. In Saeki (2006) can be found a work concerning robust PID design via LMIs.

\footnotetext{
* The authors want to thank the MICINN for funding this work through project DPI2010-19154.
}

In this work, the PID controllers have been synthesized through affine parametrization. This methodology can cancel the stable-known dynamics of the system. And for the unknown dynamics and parameter uncertainty, a robust design using $H_{\infty}$ theory is implemented. By combining robust and affine approaches, PID controllers are obtained which guarantee functionality under uncertain conditions.

The remainder of the paper is organized as follows. Section 2 describes the model of quadrotor used in this work. In Section 3, the controllers structure and its implementation are explained. Simulation results are presented in Section 4, and finally the main conclusions are drawn in Section 5.

\section{SYSTEM MODELING}

A scheme of the system is presented in Fig. 1, wherein different forces and torques actuating on the quadrotor can be observed. Basically, they are the followings: total force, $F$, roll torque, $\tau_{\phi}$, pitch toque, $\tau_{\theta}$, and yaw torque, $\tau_{\psi}$. However the actuators are four rotors represented by their forces, $\mathrm{f}_{i}$. Therefore, the desired total force and torques have to be achieved by exerting some combination of $f_{i}$ as follows:

$$
\begin{gathered}
F=\mathrm{f}_{1}+\mathrm{f}_{2}+\mathrm{f}_{3}+\mathrm{f}_{4} \\
\tau_{\phi}=l \cdot\left(\mathrm{f}_{2}-\mathrm{f}_{4}\right), \tau_{\theta}=l \cdot\left(\mathrm{f}_{3}-\mathrm{f}_{1}\right) \\
\tau_{\psi}=\frac{k_{t}}{b} \cdot\left(\mathrm{f}_{1}+\mathrm{f}_{3}-\mathrm{f}_{2}-\mathrm{f}_{4}\right),
\end{gathered}
$$

where $l$ is the distance from the center of rotation to the rotors, $k_{t}$ is the drag coefficient of the motors and $b$ is the thrust coefficient of the rotors. The following values have been considered for this work from a particular quadrotor:

$$
\begin{gathered}
k_{t}=1.06 \cdot 10^{-8} N m^{2}\left(\tau_{\psi}=k_{t} \omega^{2}\right) \\
b=5.8 \cdot 10^{-7} N s^{2}\left(\mathrm{f}=b \omega^{2}\right) .
\end{gathered}
$$

The aim is to control the height and the stabilization of the UAV simultaneously. Next, the nonlinear equations of 


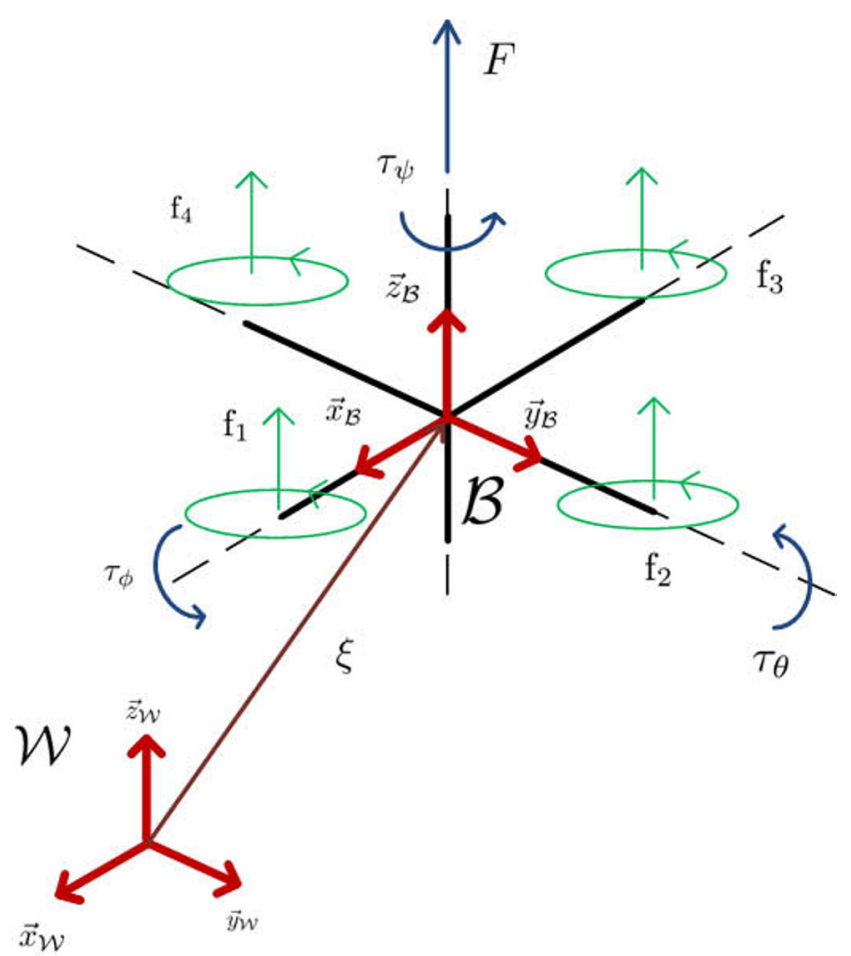

Fig. 1. Model scheme: Main forces and torques

motion for the height, $z$ and for these three angles are shown, wherein the roll, pitch and yaw Euler angles are noted as $\phi, \theta$ and $\psi$ respectively (Raffo et al (2010)), (Raffo et al (2008)).

- Equation for the height expressed with respect to an inertial frame $\mathcal{W}$ :

$$
\ddot{z}=-g+\frac{1}{m}(\cos \theta \cos \phi) F+\frac{A_{z}}{m},
$$

being $A_{z}$ a disturbance acting on the $Z$ axis. ç

- Equations for the three Euler angles:

$$
I(\eta) \ddot{\eta}+\mathbf{C}(\eta, \dot{\eta}) \dot{\eta}=\tau_{\eta}+\tau_{\eta_{\text {dist }}} .
$$

Being $I(\eta)$ inner matrix, $\eta$ Euler angles, C Coriolis matrix, $\tau_{\eta}$ torques applied and $\tau_{\eta_{\text {dist }}}$ disturbance torques.

$$
\begin{aligned}
& \tau_{\eta}=\left[\begin{array}{c}
\tau_{\phi} \\
\tau_{\theta} \\
\tau_{\psi}
\end{array}\right] \\
& \mathcal{I}(\eta)=\left[\begin{array}{lll}
i_{11} & i_{12} & i_{13} \\
i_{21} & i_{22} & i_{23} \\
i_{31} & i_{32} & i_{33}
\end{array}\right] \\
& i_{11}=I_{x x}, i_{13}=-I_{x x} \sin \theta \\
& i_{12}=i_{21}=0 \\
& i_{22}=I_{y y} \cos ^{2} \phi+I_{z z} \sin ^{2} \phi \\
& i_{23}=\left(I_{y y}-I_{z z}\right) \cos \phi \sin \phi \cos \theta \\
& i_{31}=-I_{x x} \sin \theta, i_{32}=\left(I_{y y}-I_{z z}\right) \cos \phi \sin \phi \cos \theta \\
& i_{33}=I_{x x} \sin ^{2} \theta+I_{y y} \sin ^{2} \phi \cos ^{2} \theta+I_{z z} \cos ^{2} \phi \cos ^{2} \theta \\
& \mathbf{C}(\eta, \dot{\eta})=\left[\begin{array}{lll}
c_{11} & c_{12} & c_{13} \\
c_{21} & c_{22} & c_{23} \\
c_{31} & c_{32} & c_{33}
\end{array}\right]
\end{aligned}
$$

$$
\begin{gathered}
c_{11}=0 \\
c_{12}=\left(I_{y y}-I_{z z}\right)\left(\dot{\theta} \cos \phi \sin \phi+\dot{\psi} \sin ^{2} \phi \cos \theta\right) \\
+\left(I_{z z}-I_{y y}\right) \dot{\psi} \cos ^{2} \phi \cos \theta-I_{x x} \dot{\psi} \cos \theta \\
c_{13}=\left(I_{z z}-I_{y y}\right) \dot{\psi} \cos \phi \sin \phi \cos ^{2} \theta \\
c_{21}=\left(I_{z z}-I_{y y}\right)\left(\dot{\theta} \cos \phi \sin \phi+\dot{\psi} \sin ^{2} \phi \cos \theta\right) \\
+\left(I_{y y}-I_{z z}\right) \dot{\psi} \cos ^{2} \phi \cos \theta+I_{x x} \dot{\psi} \cos \theta \\
c_{22}=\left(I_{z z}-I_{y y}\right) \dot{\phi} \cos \phi \sin \phi \\
c_{23}=-I_{x x} \dot{\psi} \sin \theta \cos \theta+I_{y y} \dot{\psi} \sin ^{2} \phi \cos \theta \sin \theta \\
+I_{z z} \dot{\psi} \cos { }^{2} \phi \sin \theta \cos \theta \\
c_{31}=\left(I_{y y}-I_{z z}\right) \dot{\psi} \cos { }^{2} \theta \sin \phi \cos \phi-I_{z z} \dot{\theta} \cos \theta \\
c_{32}=\left(I_{z z}-I_{y y}\right)\left(\dot{\theta} \cos \phi \sin \phi \sin \theta+\dot{\phi} \sin ^{2} \phi \cos \theta\right) \\
+\left(I_{y y}-I_{z z}\right) \dot{\phi} \cos { }^{2} \phi \cos \theta+I_{x x} \dot{\psi} \sin \phi \cos \theta \\
-I_{y y} \dot{\psi} \sin ^{2} \phi \sin \theta \cos \theta-I_{z z} \dot{\psi} \cos { }^{2} \phi \sin \theta \cos \theta \\
c_{33}=\left(I_{y y}-I_{z z}\right) \dot{\phi} \cos \phi \sin \phi \cos ^{2} \theta-I_{x x} \dot{\theta} \sin { }^{2} \phi \cos \theta \sin \theta \\
-I_{z z} \dot{\theta} \cos ^{2} \phi \cos \theta \sin \theta+I_{x x} \dot{\theta} \cos \theta \sin \theta
\end{gathered}
$$

These equations describe the system behavior in all its workspace. However, in this paper a hovering flight is considered, and therefore, the desired equilibrium point consists in keeping null values for the Euler angles, at least for the pitch and roll angles. Keeping in mind this fact, the original model can be simplified, yielding the following linear expressions:

$$
\ddot{z}=\frac{F}{m}, \ddot{\phi}=\frac{\tau_{\phi}}{I_{x x}}, \ddot{\theta}=\frac{\tau_{\theta}}{I_{y y}}, \ddot{\psi}=\frac{\tau_{\psi}}{I_{z z}}
$$

The values of the mass, the length from the center of the mass to the rotor and the inertias considered can be observed below, they would be obtained experimentally:

$$
\begin{gathered}
m=2.24 \mathrm{~kg}, l=0.332 \mathrm{~m}, I_{x x}=0.038425 \mathrm{kgm}^{2} \\
I_{y y}=0.038421 \mathrm{kgm}^{2}, I_{z z}=0.061547 \mathrm{kgm}^{2}
\end{gathered}
$$

Then the plant without considering the rotors dynamics is given by:

$$
G_{i}(s)=\frac{K_{i}}{s^{2}},\{i=z, \phi, \theta, \psi\}
$$

These transfer functions represent a simplified linear model obtained from movement equations. Nevertheless in this model, neither the delays from communication (of about $20 \mathrm{~ms}$ ) due to the motors driver nor motors dynamics are considered. Besides, some coupling is not considered either, which appears when the helicopter is working far from the desired equilibrium points. All these facts will be dealt as a system uncertainties.

\section{CONTROLLER DESIGN}

Four controllers are needed, one for the height and one for each angle. A PID based on affine parametrization is going to be presented. This classic controller, $C(s)$, has the following structure:

$$
C_{P I D}(s)=K_{P} \frac{T_{I} T_{D} s^{2}+T_{I} s+1}{T_{I} s\left(\tau_{D} s+1\right)},
$$

Based on an affine parametrization, the controller can be expressed in the following form (Goodwin et al (2000):

$$
C(s)=\frac{Q(s)}{1-Q(s) G(s)},
$$


where the transfer function $Q(s)$ acts as parameter which may cancel the stable dynamics of the plant. Therefore, $Q(s)$ can be expressed as follows:

$$
Q(s)=F_{Q}(s) G^{-1}(s),
$$

where $F_{Q}(s)$ is chosen to satisfy some constraints (Goodwin et al (2000)).

In the case under consideration,

$$
G^{-1}(s)=\frac{s^{2}}{K}
$$

It is known that $Q(s)$ must be proper, which yields $F_{Q}(s)$ needs to have relative degree 2 . In addition, since a specific PID structure for the controller is desired, and taking into account that input sensitivity transfer function

$$
S_{i o}(s)=\left[1-Q(s) G_{0}(s)\right] G_{0}(s)
$$

must have a zero at the origin in order to be able to reject sustained disturbances, the following general expression for $F_{Q}(s)$ is proposed:

$$
F_{Q}(s)=\frac{\alpha_{2} s^{2}+\alpha_{1} s+1}{\alpha_{4} s^{4}+\alpha_{3} s^{3}+\alpha_{2} s^{2}+\alpha_{1} s+1}
$$

By substituting this expression of $F_{Q}(s)$ into (15) and (14), and after some manipulations, the following controller is obtained:

$$
C(s)=\frac{\alpha_{2} s^{2}+\alpha_{1} s+1}{\alpha_{3} K s\left(\frac{\alpha_{4}}{\alpha_{3}} s+1\right)}
$$

By comparing the preceding expression with Eq. (13), it is easy to obtain the following correlations:

$$
K_{P}=\frac{\alpha_{1}}{\alpha_{3} K}, T_{I}=\alpha_{1}, T_{D}=\frac{\alpha_{2}}{\alpha_{1}}, \tau_{D}=\frac{\alpha_{4}}{\alpha_{3}}
$$

In this work, the following conditions have been taken into account for the controllers design. On the one hand, in order to optimize the disturbances rejection, the following constraints has been imposed:

$$
T_{I}=4 T_{D} \rightarrow \alpha_{2}=\frac{\alpha_{1}^{2}}{4}
$$

On the other hand, the time constant of the high frequency pole is chosen as follows:

$$
\tau_{D}=\frac{T_{D}}{10} \rightarrow \alpha_{4}=\frac{\frac{\alpha_{2}}{\alpha_{1}}}{10} \alpha_{3}
$$

Under these constraints, the controllers have two parameters to adjust, $\alpha_{1}$ and $\alpha_{3}$. Their adjustment will be carried out in order to provided robustness to the controlled system.

For the design of a robust controller, first the following uncertain plant is considered:

$$
G_{i}^{*}(s)=\frac{K_{i}^{*}}{s^{2}\left(\tau_{m} s+1\right)} e^{-L s}
$$

In this uncertain plant, structural and parametric uncertainties with respect to the nominal model are included. $\tau_{m}$ represents the motor time constant, whose value has been estimated about 0.1 seconds, and $L$ is the delay due to the ESC (Electronic Speed Controller) driver, whose

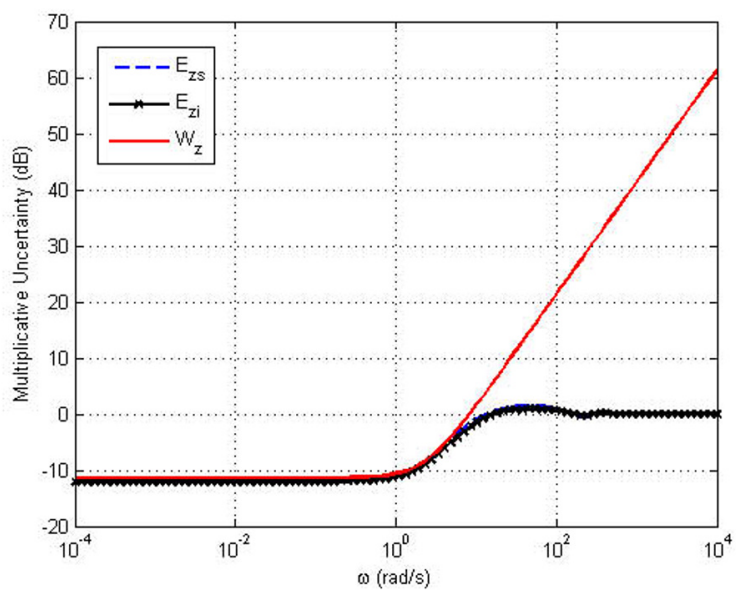

Fig. 2. Uncertainties in $\mathrm{Z}$ coordinate and estimated bound

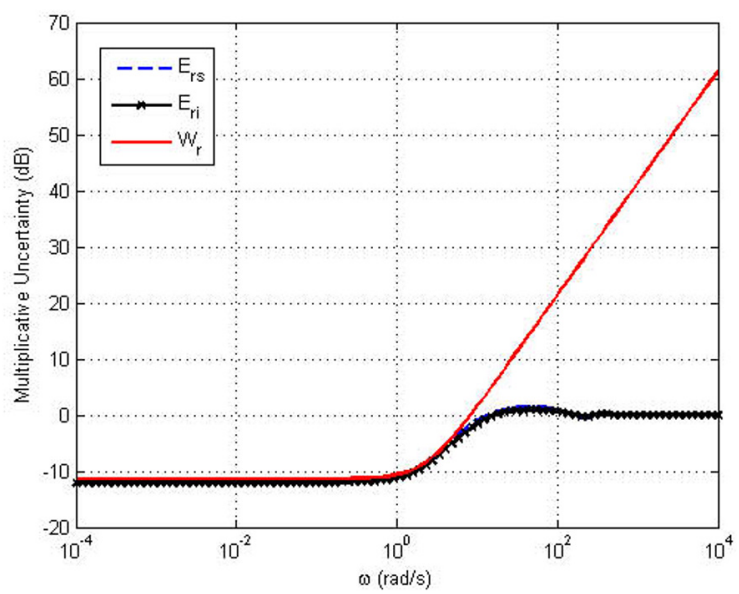

Fig. 3. Uncertainties in Roll coordinate and estimated bound

value is around $20 \mathrm{~ms}$. In addition, a gain variation of a $\pm 25 \%$ is also considered.

Once the uncertain plants have been selected, the multiplicative uncertainty with respect the nominal model can be estimated as follows:

$$
\left|E_{m i}(j \omega)\right|=\frac{\left|G_{i}^{*}(j \omega)-G_{i}(j \omega)\right|}{\left|G_{i}(j \omega)\right|},
$$

The modulus of the output multiplicative uncertainty can be upper bounded by the modulus of a stable weighting function $W_{i}(s)$. Both the estimated multiplicative uncertainty and the designed weighting function are depicted in Figs. 2 and 3 for the case of the height and the roll angle respectively. The ones for the remaining Euler angles are very similar to the roll angle case. It should be highlighted the fact that the modulus of the weighting function grows at high frequencies, which is due to the fact that the uncertain models do not represent the real plant at these frequencies.

It is well-known (Sigurd (2005)) that to achieve a robust $H_{\infty}$ controller, it is necessary the inverse of the multiplicative uncertainty bound has to be an upper bound for the complementary sensitivity transfer function $T_{i}(s)$ 


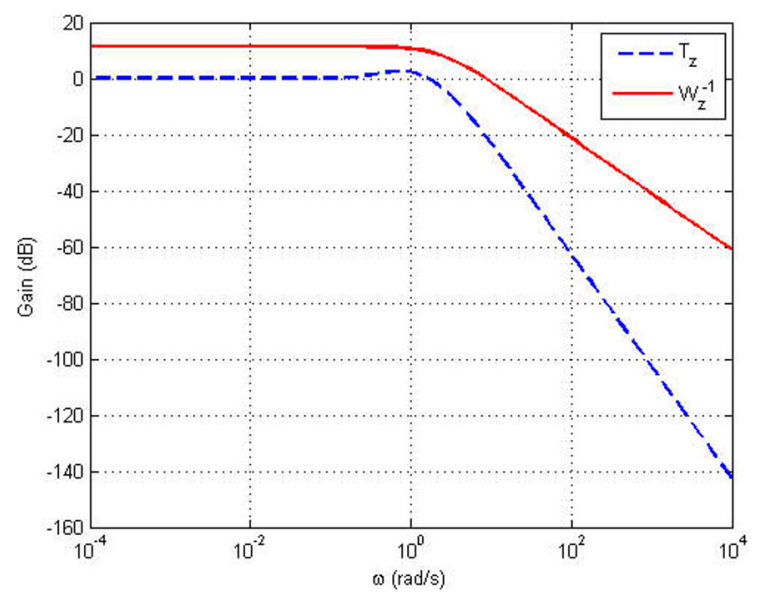

Fig. 4. Complementary sensitivity function for $\mathrm{Z}$ coordinate and its upper bound

$$
T_{i}(s)=\frac{G_{i}(s) C_{i}(s)}{1+G_{i}(s) C_{i}(s)} .
$$

for all frequencies. Consequently, the two parameters, $\alpha_{1}$ and $\alpha_{3}$, are designed in such a way that complementary sensitivity function be under the inverse multiplicative uncertainty bound and to satisfy that bandwidth be as higher as possible.

By substituting the expressions (12) and (19) into (25), the following expression for the complementary sensitivity function is obtained:

$$
T_{i}(s)=\frac{10 \alpha_{i 1}^{2} s^{2}+40 \alpha_{i 1} s+40}{\alpha_{i 1} \alpha_{i 3} s^{4}+40 \alpha_{i 3} s^{3}+10 \alpha_{i 1}^{2} s^{2}+40 \alpha_{i 1} s+40}
$$

Therefore, the coefficients $\alpha_{1 i}$ and $\alpha_{3 i}, i=z, \phi, \theta, \psi$, must be computed in order to make the corresponding $T_{i}$ be under its upper bound. The values for these coefficients designed for this application are the following:

$$
\begin{gathered}
\alpha_{z 1}=8, \alpha_{z 3}=11.2 \\
\alpha_{i 1}=5.8, \alpha_{i 3}=1.12 \quad i=\phi, \theta, \psi
\end{gathered}
$$

Figures 4 and 5 show the complementary sensitivity functions obtained whit these values for the case of the height and the roll angle, as example for the triplet of angles, respectively.

\section{SIMULATION RESULTS}

In this section, simulation results are presented wherein several step signals are applied as a reference for the triplet of angles, as well as for the height. These kind of references will force the helicopter to move on the $\mathrm{XY}$ plane without a predefined trajectory, although in any case, these movements will not be considered in this work. Therefore, it can be supposed that the quadrotor is attached to a telescopic arm to prevent such movements.

Besides, a more realistic model than the one presented in Section 2 has been used for the simulation. This model consider, among other factors, a misalignment between the mass center and the geometric center of the helicopter.

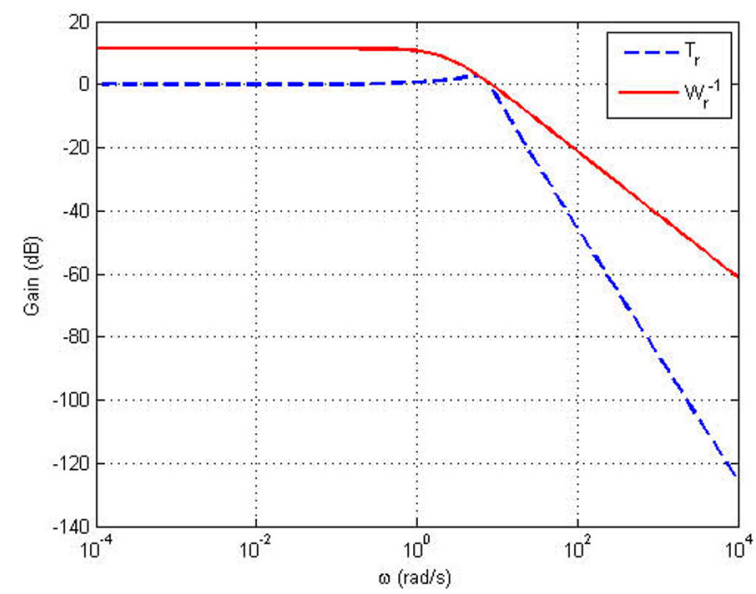

Fig. 5. Complementary sensitivity function for Roll coordinate and its upper bound

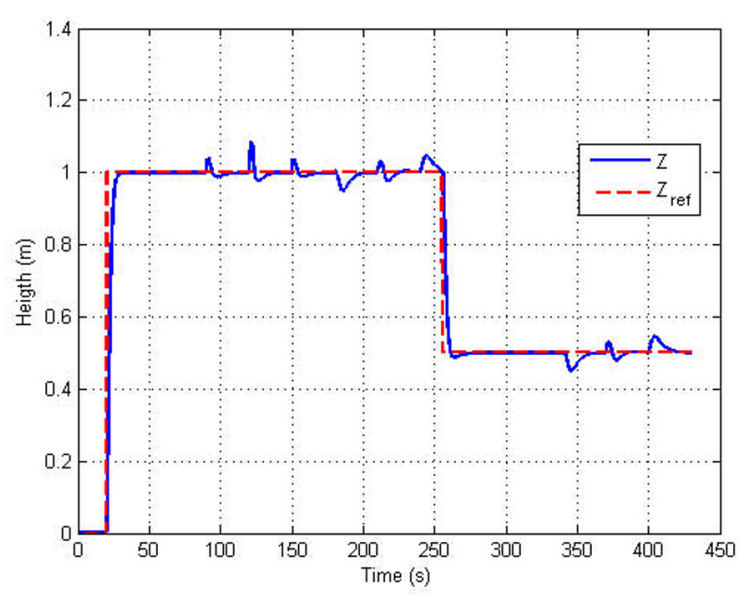

Fig. 6. Time evolution of the $\mathrm{Z}$ coordinate

The controller has been implemented in discrete time, with a sample time of $20 \mathrm{~ms}$, which simulates the time required by ESC.

The behavior of the height and the triplet of angles, roll, pitch and yaw, are shown in Fig. 6, 7, 8 and 9. The coupling effects of the system can be seen in these figures. For example, it can be seen a large variation in the roll angle at $\mathrm{t}=120 \mathrm{~s}$ due to a big reference change for the yaw angle.

Nevertheless, the slope of the step produces an overshoot. To mitigate that effect, a first-order filter for the references has been designed, whose time constant has been selected to counteracts the controller zeros. Figures 10, 11, 12, 13 show how the behavior improves with the filter. In can be seen that the benefits from the filter addition are double. On the one hand, the overshoots of the step response disappear but the time response gets slower. On the other hand, this last fact makes that a step change in reference of one coordinate affects in a smoother way on the others coordinates, which improves the decoupling of the controller system.

Finally, it can be noticed by comparing Figs. 4 and 5 that the control bandwidth for the height is not as high as the one for the angles. However, although the bandwidth 


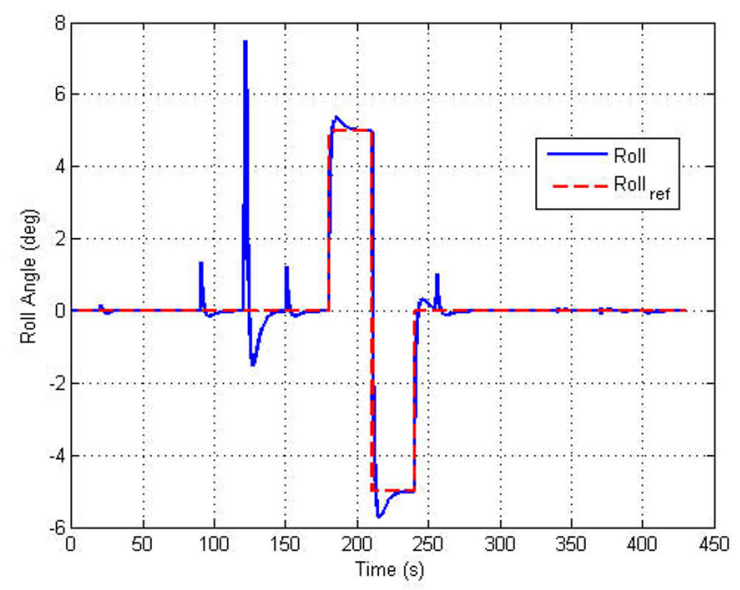

Fig. 7. Time evolution of the Roll angle

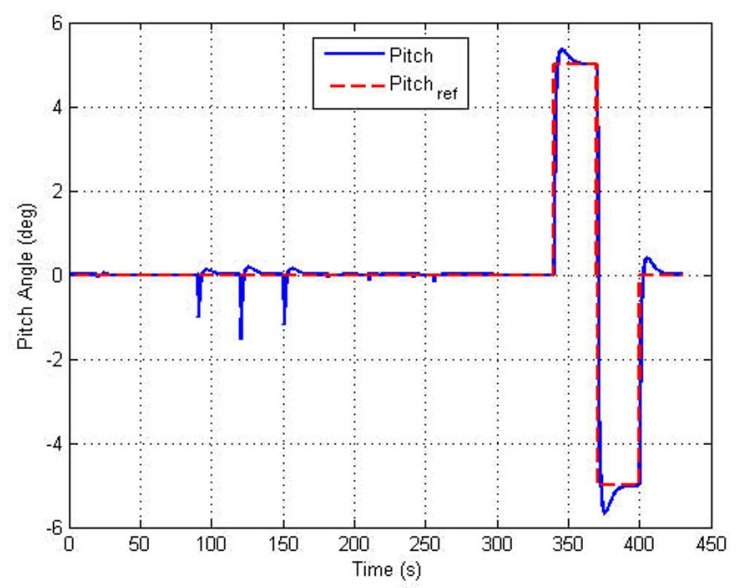

Fig. 8. Time evolution of the Pitch angle

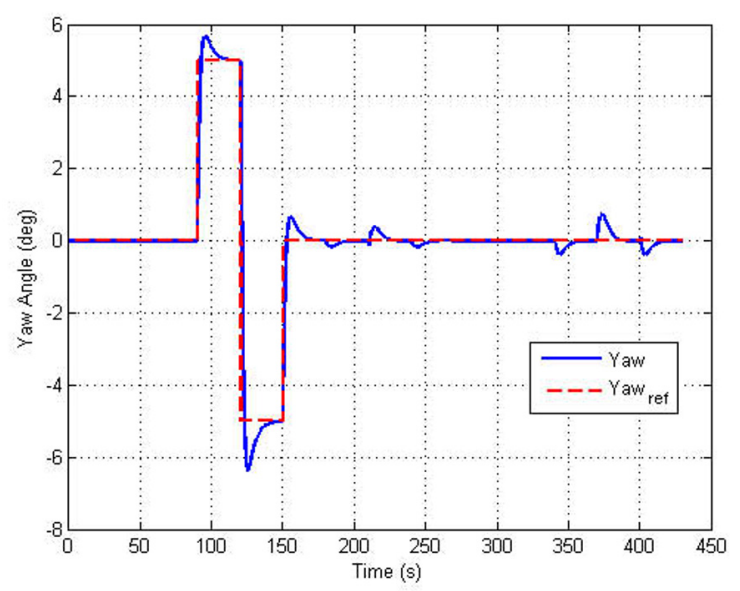

Fig. 9. time evolution of the Yaw angle

control for the z coordinate may be increased, this fact may cause undesirable effects due to the coupling. As for example, Figs. 14 and 15 show the behavior of the $\mathrm{Z}$ and roll coordinates for the case of the same control bandwidth for all coordinates, which is achieved by selecting the same values for $\alpha_{z 1}$ and $\alpha_{z 3}$ than the ones for $\alpha_{\phi 1}$ and $\alpha_{\phi 3}$ respectively. Obviously, the control of the height gets

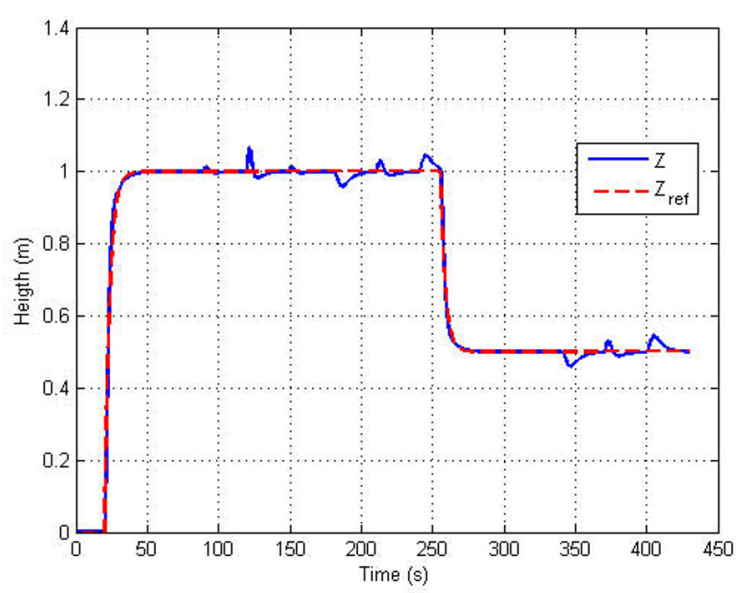

Fig. 10. Time evolution of the $\mathrm{Z}$ coordinate with filtered reference

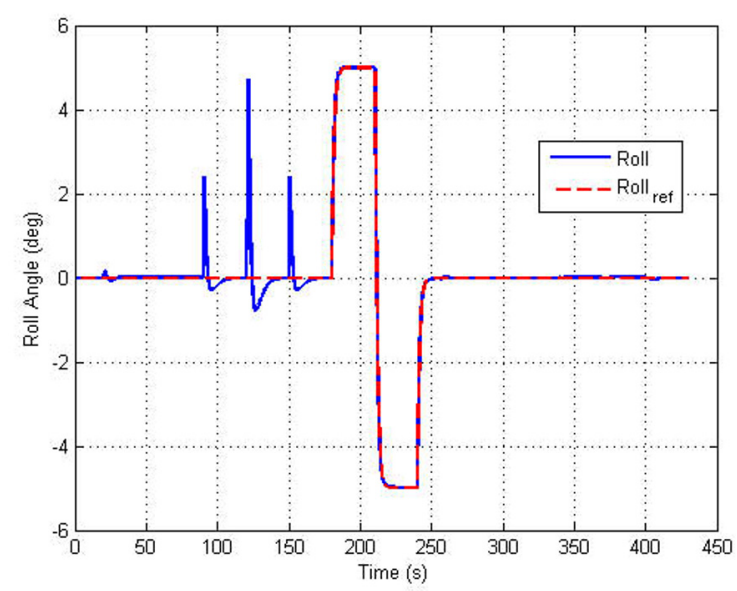

Fig. 11. Time evolution of the Roll angle with filtered reference

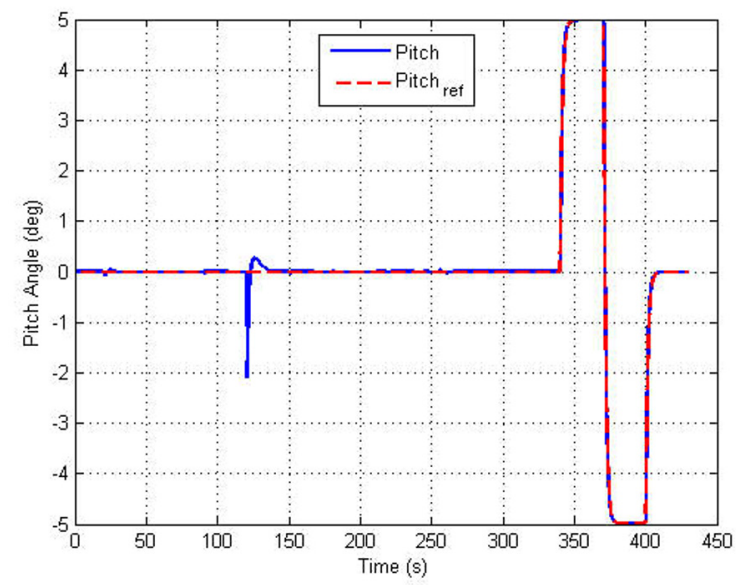

Fig. 12. Time evolution of the Pitch angle with filtered reference 


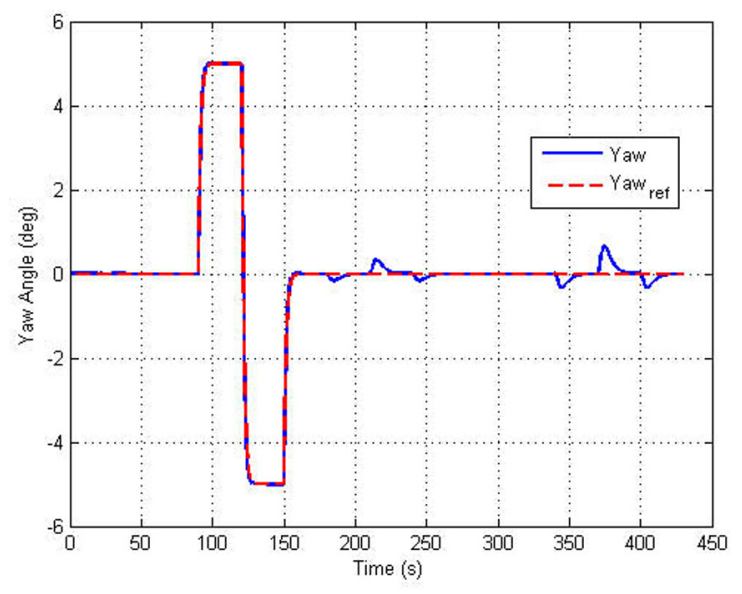

Fig. 13. Time evolution of the Yaw angle with filtered reference

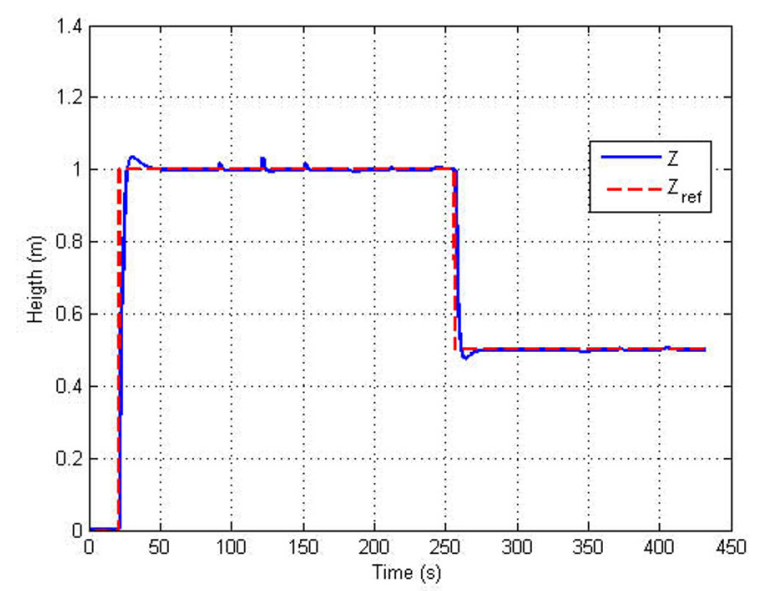

Fig. 14. Time evolution of the $\mathrm{Z}$ coordinate with high height-control bandwidth

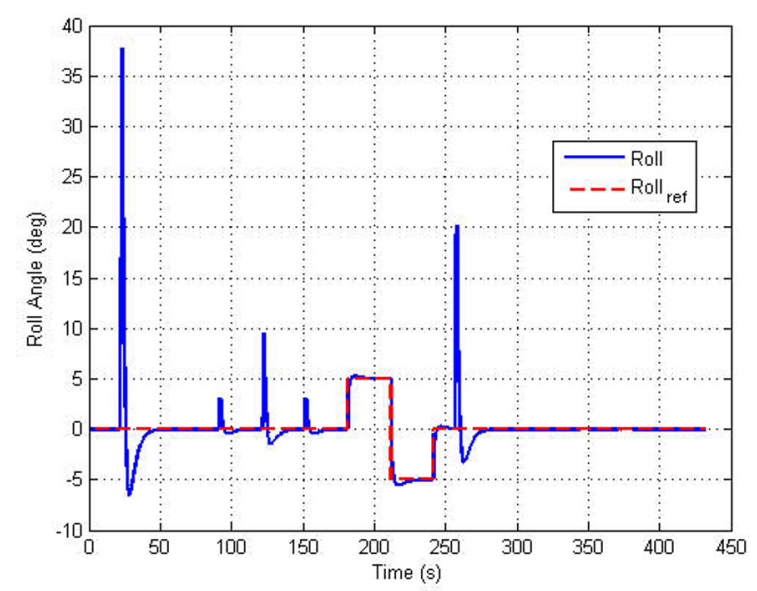

Fig. 15. Time evolution of the Roll angle with high heightcontrol bandwidth

better, but a reference change in $\mathrm{Z}$ affects in excess on the performance of the angles. This is clearly undesirable for future works where the displacements in the XY plane have to be considered.

\section{CONCLUSION}

In this paper a robust PID control strategy, tuned via affine parametrization, has been presented. At first, a decoupled system has been assumed, but in figures of simulation results it is observed that as long as a reference angle changes, the other ones can be modified. This is because the equations used in the simulation model are coupled, whereas the equations used for control are decoupled.

It can be seen how the behavior of the system is quite good even with the simplifications made. So a nonlinear system can be controlled with a decoupled linear controller. This controller is a PID tuning by affine parametrization and robust thanks to $H_{\infty}$ theory.

Affine parametrization allows to cancel some dynamics in order to obtain a desired dynamics. In addition, $H_{\infty}$ theory allows to considerer uncertainties such as the plant with different gain and the actuator, motors in this case.

However it could be observed an overshoot due to the applied reference. To solve that a filter in the reference was used and the results obtained were better and slower.

\section{REFERENCES}

K. Alexis, G. Nikolakopoulos and A. Tzes Model Predictive Control Scheme for the Autonomous Flight of an Unmanned Quadrotor Industrial Electronics (ISIE), 2011 IEEE International Symposium on. 2243-2248, 2011

E. Altug, J.P. Ostrowski, R. Mahony Control of a Quadrotor Helicopter Using Visual Feedback Robotics and Automation, 2002. Proceedings. ICRA '02. IEEE International Conference on Vol.1 72 - 77, 2002

P. Castillo, P. Garcia, R. Lozano, P. Albertos. Modelado y Estabilización de un Helicóptero con cuatro rotores. Revista Iberoamericana de Automática e Informática Industrial RIAI, ISSN: 1697-7912 4:41-57, 2007.

M. Chen, M. Huzmezan A Combined MBPC/2 DOF $H_{\infty}$ Controller for a Quad Rotor UAV Proc. AIAA Guidance, Navigation, and Control Conference and Exhibit Texas, USA, 2003.

M. Chen, M. Huzmezan A Simulation Model and $H_{\infty}$ Loop shaping Control of a Quad Rotor Unmanned Air Vehicle Proceedings of Modelling, Simulation, and Optimization, 320-325, 2003

G. C. Goodwin, S. F. Graebe, M. E. Salgado. Control System Design. Valparíso, 2000 .

M. Saeki Fixed structure PID controller design for standar $H_{\infty}$ control problem. Automatica, 42:93-100, 2006.

G. V. Raffo, M. G. Ortega, F. R. Rubio An integral predictive/nonlinear $H_{\infty}$ control structure for a quadrotor helicopter Automatica, 46:29-39, 2010.

G. V. Raffo, M. G. Ortega, F. R. Rubio Robust Backstepping/Nonlinear $H_{\infty}$ control for path tracking of a quadrotor unmanned aerial vehicle. Proceedings American Control Conference (Acc'08) Seattle. Wa. Usa, 2008.

S. Skogestad and I. Postlethwaite Multivariable Feedback Control Analysis and Design. John Wiley and Sons Ltd, 2005 